\title{
Neutral and charged excitons in a quantum tube
}

\author{
Shintaro Nomura* ${ }^{*}, 2$ and Kohei Tsumura ${ }^{1}$ \\ ${ }^{1}$ Institute of Physics, University of Tsukuba, Tennodai, Tsukuba, Ibaraki, Japan. \\ ${ }^{2}$ CREST-JST, 4-1-8 Hon-cho, Kawaguchi, Japan. \\ (Received 28 June 2006)
}

The optical transition energies of neutral and charged excitons in a quantum tube are calculated as a function of the Aharonov-Bohm magnetic flux $\Phi$. The oscillation amplitude of the ground state energy of the electron-hole relative motion is shown to be larger in a quantum tube than a quantum ring with strong confinement in the axis direction. We find a double maxima structure in the optical transition energy for a quantum tube with radius $R=0.5$ in units of the effective Bohr radius because of the difference in the $\Phi$ dependencies between the single electron energy and the relative motion energy of a charged exciton state.

Keywords: Aharonov-Bohm effect, quantum tube, photoluminescence, charged exciton 


\section{Introduction}

The Aharonov-Bohm (AB) effect of an electron-hole composite system has recently been actively investigated after a theoretical analysis of $\mathrm{AB}$ oscillation of the energy of a neutral exciton $\left(\mathrm{X}^{0}\right)$ on a quantum ring [1,2]. The $\mathrm{AB}$ effect of electrons is described by single-particle property of a charged particle coupled to vector potential. The ground state energy of the electron changes periodically with the magnetic flux penetrating the ring divided by the magnetic flux quantum $\phi_{0}=c h / e$. In the excitonic $\mathrm{AB}$ effect, the relativemotion of an electron and a hole which couple to vector potential gives the oscillation of the energy. The center-of-mass motion of a neutral exciton shows no sensitivity to vector potential. The amplitude of $\mathrm{AB}$ oscillation depends critically on the size of the exciton, which is determined by the electron-hole Coulomb correlation.

There have been several attempts to observe the excitonic AB effect of an electron-hole composite. The oscillations of the photoluminescence (PL) peak energy from a charged exciton $\left(\mathrm{X}^{-}\right)[3,4]$ and from a type-II exciton [5] with magnetic fields were observed in samples with ring geometries. However, the observed oscillations were explained by single particle energy of an electron or a hole.

This difficulty in the observation of the excitonic $A B$ effect originating from the relative-motion of an electron and a hole is inherent to a ring-geometry. A quantum ring is one-dimensional (1D) system with a periodic boundary condition. It is known that the binding energy of an electron-hole pair in 1D diverges if a bare Coulomb potential is assumed [6,7]. Therefore, a cutoff parameter is often introduced in the Coulomb interaction term to avoid the divergence. While the potential confinement strength is finite in actual samples, the strong electron-hole Coulomb correlation in quasi-1D system nevertheless leads to a small effective Bohr radius and thus small amplitude of $\mathrm{AB}$ oscillation of the ground state energy, which makes it difficult to observe $\mathrm{AB}$ oscillation due to the relative motion.

A prescription for enhancing the excitonic $\mathrm{AB}$ effect is to remove the potential confinement parallel to the axis of a ring, that is, to use a quantum tube. This additional freedom of motion reduces the electron-hole Coulomb correlation, which enhances the coupling of the relative motion of an electron and a hole to vector potential. Moreover, in a 
quantum tube it is not necessary to introduce any artificial cutoff parameter for the Coulomb potential theoretically because we deal with 2-dimensional (2D) system. In this paper, we investigate the excitonic $\mathrm{AB}$ effect in a quantum tube with isotropic, nondegenerate, and parabolic conduction and valence bands in the effective-mass approximation. The AB effect in carbon nanotubes were investigated in the effective-mass approximation by solving Weyl's equation [8-11].

The purpose of the present paper is to show that a quantum tube is more favorable to observe the excitonic $\mathrm{AB}$ effect than a quantum ring with the same radius. We investigate both the cases of $\mathrm{X}^{0}$ and $\mathrm{X}^{-}[4,12-16]$. The oscillation of the optical transition energies is investigated as a function of magnetic flux quantum. This paper is motivated by a recent report on successful growth of quantum tube structures [17].

\section{Method of calculation}

\subsection{Neutral exciton}

A quantum tube with radius $R$ in magnetic field parallel to the axis of the tube $\left(\mathbf{e}_{\mathrm{z}}\right)$ is modeled in the effective-mass approximation. The motion of electrons at coordinates $\mathbf{r}_{\mathbf{j}}^{(e)}$ $(\mathrm{j}=1,2)$ and a hole at coordinate $\mathbf{r}^{(h)}$ in the magnetic flux $\Phi$ is considered. The electrons and the hole are confined in a static potential, interacting by the Coulomb interaction $V_{\text {e-e }}(\mathbf{r})$ and $V_{\text {e-h }}(\mathbf{r})$. The Hamiltonian can be separated into the center-of-mass motion $\left(H_{\mathrm{c} . \mathrm{m} .}\right)$ and the relative motion with respect to a hole $\left(H_{\text {rel }}\right)$ following Ref. $[4,12]$. A cylindrical coordinate $\mathbf{r}_{\mathrm{j}}=\left(r_{\mathrm{j}}, \varphi_{\mathrm{j}}, z_{\mathrm{j}}\right)$ is used and we choose the $z_{\mathrm{j}}$-axis in the axis direction and $\varphi_{\mathrm{j}}$ axis in the circumference direction. In the case of $\mathrm{X}^{0}$, the Hamiltonian is given by

$H^{\left(\mathrm{X}^{0}\right)}=H_{\mathrm{c} . \mathrm{m} .}^{\left(\mathrm{X}^{0}\right)}+H_{\mathrm{rel}}^{\left(\mathrm{X}^{0}\right)}$,

$H_{\mathrm{c} . \mathrm{m} .}^{\left(\mathrm{X}^{0}\right)}=-\frac{\hbar^{2}}{2\left(m_{e}+m_{h}\right)} \nabla_{\mathrm{c} . \mathrm{m} .}^{2}$

and

$H_{\mathrm{rel}}^{\left(\mathrm{X}^{0}\right)}=\frac{\hbar^{2}}{2 \mu^{*}}\left(\frac{\nabla_{r e l}}{i}+\frac{\Phi}{R \phi_{0}} \mathbf{e}_{\varphi}\right)^{2}+V_{e h}\left(\mathbf{r}_{r e l}\right)$, 
where

$$
z_{r d}=\mathrm{z}^{(e)}-\mathrm{Z}^{(h)},
$$$$
z_{\mathrm{c} . \mathrm{m} .}^{\left(\mathrm{X}^{0}\right)}=\frac{m_{e}}{m_{e}+m_{h}} z^{(e)}+\frac{m_{h}}{m_{e}+m_{h}} z^{(h)},
$$

$\varphi_{r e l}=\varphi^{(e)-\varphi^{(h)}}, \varphi_{\mathrm{c} . \mathrm{m} .}^{\left(\mathrm{X}^{0}\right)}=\frac{m_{e}}{m_{e}+m_{h}} \varphi^{(e)}+\frac{m_{h}}{m_{e}+m_{h}} \varphi^{(h)}, \mu^{*-1}=m_{\mathrm{e}}^{-1}+m_{\mathrm{h}}{ }^{-1}, \mathbf{e}_{\varphi}$ is a unit vector in the circumference direction, $m_{\mathrm{e}}$ and $m_{\mathrm{h}}$ are the effective-masses of an electron and a hole, respectively. The electron-hole Coulomb interaction term is given by

$$
V_{e-h}\left(\mathbf{r}_{1}\right)=-\frac{e^{2}}{\varepsilon \sqrt{z_{1}^{2}+4 R^{2} \sin ^{2}\left(\varphi_{1} / 2\right)}}
$$

where the static dielectric constant $\varepsilon$ is assumed to be the same for the inside and the outside of the tube. An infinitesimally thin quantum tube is assumed, i.e., $r_{\mathrm{j}}$ is fixed to the radius of the tube $R$. The wave function for the center-of-mass motion is given by

$$
\Psi_{K, N_{\mathrm{c} . \mathrm{m}}}^{\left(\mathrm{X}^{0}, \mathrm{~cm}\right)}\left(z_{\mathrm{c} . \mathrm{m} .}, \varphi_{\mathrm{c.m} .}\right)=A_{\mathrm{c.m} .}^{\left(\mathrm{X}^{0}\right)} \exp \left[i K z_{\mathrm{cm} . \mathrm{m} .}\right] \exp \left[i N_{\mathrm{c.m} .} \varphi_{\mathrm{c} . \mathrm{m} .}\right]
$$

where $A_{\mathrm{c} . \mathrm{m}}^{\left(\mathrm{X}^{0}\right)}$ is a normalization coefficient, $K$ is the wave number in the axis direction, and $N_{\text {c.m. }}$ is the angular momentum of the center-of-mass motion. $\Psi_{K, N_{\text {c.m }}}^{\left(\mathrm{X}^{0}, \text { c.m. }\right.}\left(z_{\text {c.m. }}, \varphi_{\text {c.m. }}\right)$ is a plane wave, and is not affected by the magnetic flux. The energy of the center-of-mass motion is given by

$E_{K, N_{\mathrm{cm}}}^{\left(\mathrm{X}^{0} \text { c.m. }\right.}=\frac{\hbar^{2}}{2\left(m_{e}+m_{h}\right)}\left[K^{2}+\left(\frac{N_{\mathrm{c.m} .} .}{R}\right)^{2}\right]$.

We are interested in the optically active $\mathrm{X}^{0}$ near the $\Gamma$ point and the energy of the center-ofmass motion is set to zero, i.e., $K=0$ and $N_{\text {c.m. }}=0$. The wave function for the relative motion is expanded using a lattice model in the axis direction and using a basis function $\chi_{N_{1}}\left(\varphi_{1}\right)=\exp \left(i N_{1} \varphi_{1}\right) / \sqrt{2 \pi}$ in the circumference direction as given by

$$
\Psi^{\left(X^{0}, \text { rel }\right)}\left(z_{1}, \varphi_{1}\right)=\sum_{N_{1}} A_{z_{1}, N_{1}} \chi_{N_{1}}\left(\varphi_{1}\right),
$$

where $A_{z_{1}, N_{1}}$ are coefficients to be determined by a diagonalization procedure. The kinetic energy term of the relative motion in an infinitesimally thin quantum tube is given by

$E_{\mathrm{KE}}^{\left(\mathrm{X}^{0}, \mathrm{el}\right)}\left(z_{1}, N_{1}\right)=E_{z_{1}}^{\left(\mathrm{X}^{0}\right)}+\frac{\hbar^{2}}{2 \mu^{*}}\left(\frac{N_{1}+\Phi / \phi_{0}}{R}\right)^{2}$,

where $E_{z_{1}}^{\left(\mathrm{X}^{0}\right)}$ is the kinetic energy of the motion in the axis direction and is independent of $\Phi$. $E_{\mathrm{KE}}^{\left(\mathrm{X}^{0}, \text { rel }\right)}$ is invariant by a transformation of

$\Phi \rightarrow \Phi^{\prime}=\Phi+p \phi_{0}$ 
and

$N_{1} \rightarrow N_{1}^{\prime}=N_{1}-p$,

where $p$ is an integer. Moreover, the electron-hole interaction matrix element as given by

$\left\langle\chi_{N_{2}}\left|V_{e h}\left(\mathbf{r}_{1}\right)\right| \chi_{N_{1}}\right\rangle=-\frac{e^{2}}{\varepsilon} \int_{-\pi}^{\pi} \frac{\mathrm{d} \varphi}{2 \pi} \frac{\cos \left(N_{2}-N_{1}\right) \varphi}{\sqrt{z_{1}^{2}+4 R^{2} \sin ^{2}(\varphi / 2)}}$

is a function of $N_{2}-N_{1}$ and is invariant by a transformation of $N_{1} \rightarrow N_{1}^{\prime}=N_{1}-p$ and $N_{2} \rightarrow N_{2}{ }^{\prime}=N_{2}-p$. Then, the energy of a $\mathrm{X}^{0}$ state is a periodic function $\Phi$ with a period of $\phi_{0}$.

\subsection{Charged exciton}

The Hamiltonian of a $\mathrm{X}^{-}$state is given by

$H^{(\mathrm{X})}=\sum_{j=1}^{2}\left[\frac{\hbar^{2}}{2 m_{e}}\left(\frac{\nabla_{j}^{(\mathrm{e})}}{i}+\frac{\Phi}{R \phi_{0}} \mathbf{e}_{\varphi}\right)^{2}+V_{e h}\left(\mathbf{r}_{j}^{(\mathrm{e})}-\mathbf{r}_{h}\right)\right]+\frac{\hbar^{2}}{2 m_{h}}\left(\frac{\nabla^{(\mathrm{h})}}{i}-\frac{\Phi}{R \phi_{0}} \mathbf{e}_{\varphi}\right)^{2}+V_{e e}\left(\mathbf{r}_{1}^{(\mathrm{e})}-\mathbf{r}_{2}^{(\mathrm{e})}\right)$,

where $\mathbf{e}_{\varphi}$ is a unit vector in the circumference direction. Similarly to the case of $\mathrm{X}^{0}$, the wave function can be expanded as given by

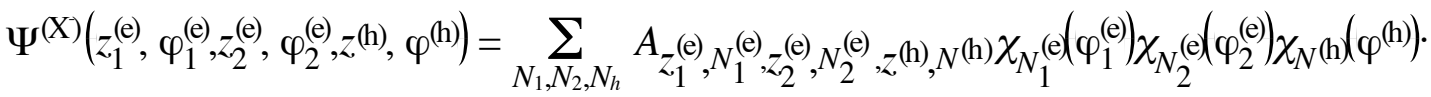

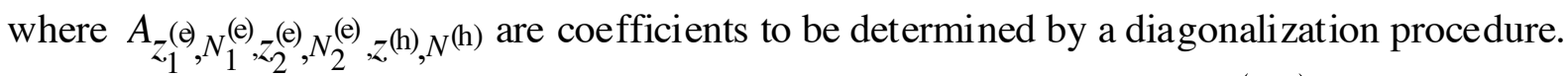
A periodic boundary condition is imposed to the basis functions $\chi_{N_{j}}(\mathrm{e})\left(\varphi_{j}^{(\mathrm{e})}\right)$ and $\chi_{N}(\mathrm{~h})\left(\varphi^{(\mathrm{h})}\right)$, which gives conditions that the quantum numbers $N_{1}^{(\mathrm{e})}, N_{2}^{(\mathrm{e})}$ and $N^{(\mathrm{h})}$ are integer numbers. The kinetic energy term is given by

$$
\begin{aligned}
E_{\mathrm{KE}}^{(\mathrm{X})}\left(z_{1}^{(\mathrm{e})}, \varphi_{1}^{(\mathrm{e})}, z_{2}^{(\mathrm{e})}, \varphi_{2}^{(\mathrm{e})}, z^{(\mathrm{h})}, \varphi^{(\mathrm{h})}\right)= & E_{z_{1}^{(\mathrm{e})}}^{(\mathrm{X}, \mathrm{e})}+E_{z_{2}^{(\mathrm{e})}}^{(\mathrm{X}, \mathrm{e})}+E_{z^{(\mathrm{h})}}^{(\mathrm{X}, \mathrm{h})} \\
& +\frac{\hbar^{2}}{2 m_{e^{-}}}\left[\left(\frac{N_{1}^{(\mathrm{e})}+\Phi / \phi_{0}}{R}\right)^{2}+\left(\frac{N_{2}^{(\mathrm{e})}+\Phi / \phi_{0}}{R}\right)^{2}\right]+\frac{\hbar^{2}}{2 m_{h}}\left(\frac{N^{(\mathrm{h})}-\Phi / \phi_{0}}{R}\right)^{2},
\end{aligned}
$$

where $E_{z_{j}^{(\mathrm{e})}}^{(\mathrm{X}, \mathrm{e})}$ and $E_{z^{(\mathrm{h})}}^{(\mathrm{X}, \mathrm{h})}$ are the kinetic energy of the motion in the axis direction for electrons and a hole, respectively, and are independent of $\Phi$. The kinetic energy term is invariant by a transformation of

$\Phi \rightarrow \Phi^{\prime}=\Phi+p \phi_{0}$

$N_{1}^{(e)} \rightarrow N_{1}^{(e)}=N_{1}^{(e)}-p$

$N_{2}^{(e)} \rightarrow N_{2}^{(e)}=N_{2}^{(e)}-p$ 
and

$N^{(\mathrm{h})} \rightarrow N_{2}^{(\mathrm{h})}=N_{2}^{(\mathrm{h})}+p$,

where $p$ is an integer. The Coulomb interaction term is calculated by evaluating the matrix element

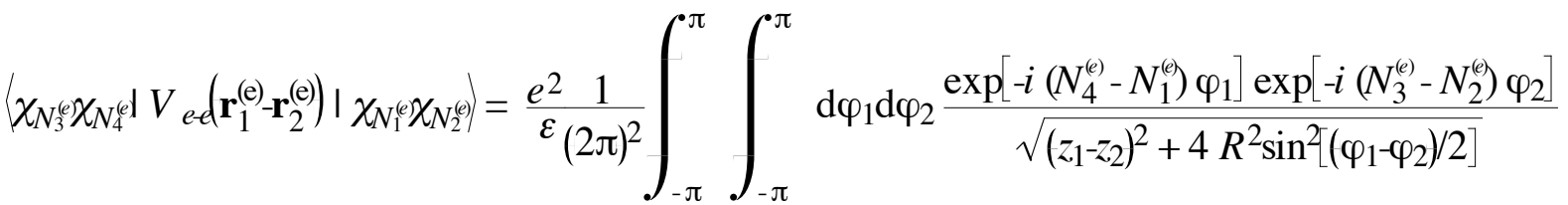

which is invariant by a transformation of (15), (16), (17), $N_{4}^{(e)} \rightarrow N_{4}^{(e){ }_{1}}=N_{4}^{(e)}-p$, and $N_{3}^{(e)} \rightarrow N_{3}^{(e){ }_{1}}=N_{3}^{(e)}-p$. Similarly, the matrix element of $V_{e-h}\left(\mathbf{r}_{j}^{(e)}-\mathbf{r}_{h}\right)$ is shown to be invariant. Then, the energy of a $\mathrm{X}^{-}$state is a periodic function $\Phi$ with a period of $\phi_{0}$.

In order to reduce the number of the basis functions, separation of a center-of-mass motion and relative-motions is introduced. The Hamiltonian is rewritten as

$H=H_{\text {c.m. }}+H_{\text {rel }}$,

$H_{\mathrm{c} . \mathrm{m} .}=\frac{\hbar^{2}}{2 M}\left(\frac{\nabla_{\mathrm{c} . \mathrm{m} .}}{i}+\frac{\Phi}{R \phi_{0}} \mathbf{e}_{\varphi}\right)^{2}$

and

$H_{\text {rel }}=\sum_{j=1}^{2}\left[\frac{\hbar^{2}}{2 \mu^{*}}\left(\frac{\nabla_{j}}{i}+\frac{\Phi}{R \phi_{0}} \mathbf{e}_{\varphi}\right)^{2}+V_{e-h}\left(\mathbf{r}_{j}\right)\right]+V_{e-d}\left(\mathbf{r}_{1}-\mathbf{r}_{2}\right)-\frac{\hbar^{2}}{m_{h}} \nabla_{1} \nabla_{2}-\frac{2 \sigma}{1+2 \sigma} \frac{\hbar^{2}}{2 \mu^{*}}\left(\frac{\Phi}{R \phi_{0}}\right)^{2}$,

where $\quad z_{j}=z_{j}^{(\mathrm{e})}-z^{(\mathrm{h})}, \quad z_{\mathrm{c} . \mathrm{m} .}=\frac{m_{e}}{M}\left(z_{1}^{(e)}+z_{2}^{(e)}\right)+\frac{m_{h}}{M} z^{(h)}, \quad \phi_{j}=\varphi_{j}^{(\mathrm{e})}-\varphi^{(\mathrm{h})}, \quad \phi_{\mathrm{c} . \mathrm{m} .}=\frac{m_{e}}{M}\left(\varphi_{1}^{(e)}+\varphi_{2}^{(e)}\right)+\frac{m_{h}}{M} \varphi^{(h)}$, $M=2 m_{\mathrm{e}}+m_{\mathrm{h}}, \sigma=m_{\mathrm{e}} / m_{\mathrm{h}}$ [4]. The wave function for the center-of-mass motion of $\mathrm{X}-$ is given by

$\Psi^{(\text {c.m. } .}\left(z_{c . m .}, \phi_{c . m .}\right)=B_{v_{c . m .}^{(c . m .)}}^{(x p}\left(i K z_{c . m}\right) \chi_{v_{c . m}}\left(\phi_{c . m}\right)$

where $K$ is the wave vector in the axis direction, $v_{\mathrm{c} . \mathrm{m}}$ is the angular momentum quantum number, $B_{v_{\mathrm{c} . \mathrm{m}}}^{\text {(c.m.) }}$ is a coefficient. The energy of the center-of-mass motion as given by

$E^{(X, c . m .)}=\frac{\hbar^{2} K^{2}}{2 M}+\frac{\hbar^{2}}{2 M R^{2}}\left(v_{c . m .}+\frac{\Phi}{\phi_{0}}\right)^{2}$

oscillates with a period of $\phi_{0}$. A periodic boundary condition is imposed to $\chi_{v_{c . m}}\left(\phi_{\mathrm{c} . \mathrm{m}}\right.$. $)$ giving that $v_{\mathrm{c} . \mathrm{m} .}$ is integer.

The wave function for the relative motion of $\mathrm{X}^{-}$is expanded as 
$\Psi^{(\mathrm{X}, \mathrm{rel})}\left(z_{1}, \phi_{1}, z_{2}, \phi_{2}\right)=\sum_{v_{1}, v_{2}} B_{z_{1}, v_{1}, z_{2}, \nu_{2}} \chi_{v_{1}}\left(\phi_{1}\right) \chi_{v_{2}}\left(\phi_{2}\right)$

where $B_{z_{1}, v_{1}, z_{2}, v_{2}}$ are coefficients to be determined by a diagonalization process. Then the total wave function is given by

$\Psi^{(\mathrm{X})}\left(z_{\text {c.m. }}, \phi_{\text {c.m. } . z_{1}}, \phi_{1}, z_{2}, \phi_{2}\right)=\sum_{v_{\mathrm{c} . \mathrm{m} .}} \Psi^{(\mathrm{c} . \mathrm{m} \cdot)}\left(z_{\mathrm{c} . \mathrm{m} .}, \phi_{\mathrm{c} . \mathrm{m} .}\right) \Psi^{(\mathrm{X} ; \mathrm{rel})}\left(z_{1}, \phi_{1}, z_{2}, \phi_{2}\right)$.

We require that this wave function should recover the form of Eq. (13) by substituting $\phi_{j}=\varphi_{j}^{(\mathrm{e})}-\varphi^{(\mathrm{h})}$, and $\phi_{\mathrm{c} . \mathrm{m} .}=\frac{m_{e}}{M}\left(\varphi_{1}^{(e)}+\varphi_{2}^{(e)}\right)+\frac{m_{h}}{M} \varphi^{(h)}$. This gives relations for $v_{1}, v_{2}$, and $v_{\mathrm{c} . \mathrm{m} .}$ as given by

$v_{1}=N_{1}^{(\mathrm{e})}-v_{\mathrm{c} \cdot \mathrm{m}} \cdot \frac{\sigma}{2 \sigma+1}$

and

$v_{2}=N_{2}^{(\mathrm{e})}-v_{\mathrm{c} \cdot \mathrm{m}} \cdot \frac{\sigma}{2 \sigma+1}$

Here $N_{1}^{(\mathrm{e})}, N_{2}^{(\mathrm{e})}$ and $v_{\mathrm{c} . \mathrm{m} \text {. }}$ are integer numbers, but $v_{1}$ and $v_{2}$ are not necessarily integer numbers depending on $\sigma$. The kinetic energy term of the relative motion is given by

$$
\begin{array}{r}
E_{\mathrm{KE}}^{(\mathrm{X}, \text { rel })}\left(z_{1}, z_{2}, v_{1}, v_{2}\right)=E_{z_{1}}^{\left(\mathrm{X}^{-}\right)}+E_{z_{2}}^{\left(\mathrm{X}^{-}\right)}+\frac{\hbar^{2}}{2 \mu^{*} R^{2}}\left[\left(v_{1}+\Phi / \phi_{0}\right)^{2}+\left(v_{2}+\Phi / \phi_{0}\right)^{2}+\frac{2 \sigma}{1+\sigma} v_{1} v_{2}-\frac{2 \sigma}{1+2 \sigma}\left(\Phi / \phi_{0}\right)^{2}\right] \\
=E_{z_{1}}^{\left(\mathrm{X}^{-}\right)}+E_{z_{2}}^{\left(\mathrm{X}^{-}\right)}+\frac{\hbar^{2}}{2 \mu^{*} R^{2}}\left\{\frac{2 \sigma}{1+2 \sigma}\left[\left(\frac{1+2 \sigma}{1+\sigma} v_{1}+\frac{\Phi}{\phi_{0}}\right)^{2}+\left(\frac{1+2 \sigma}{1+\sigma} v_{2}+\frac{\Phi}{\phi_{0}}\right)^{2}\right]-\frac{\sigma}{1+\sigma}\left(v_{1}-v_{2}\right)^{2}\right\}
\end{array}
$$

where $E_{z_{j}}^{\left(\mathrm{X}^{-}\right)}$is the kinetic energy of the motion in the axis direction. In the followings, we consider two cases: the first is the case where $\nu_{\mathrm{c} . \mathrm{m}}$ is constant in changing $\Phi$, and the second

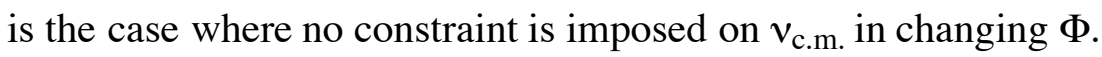

In the first case, $E_{\mathrm{KE}}^{(\mathrm{X} \text {; } \mathrm{el})}$ is invariant by a transformation of

$\Phi \rightarrow \Phi^{\prime}=\Phi+\frac{1+2 \sigma}{1+\sigma} p \phi_{0}$

$v_{1} \rightarrow v_{1}{ }^{\prime}=v_{1}-p$

and

$v_{2} \rightarrow v_{2}^{\prime}=v_{2}-p$.

The attractive and repulsive Coulomb interaction terms are written as

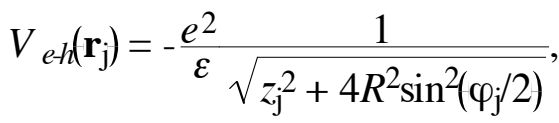


and

$V_{e-t}\left(\mathbf{r}_{1}-\mathbf{r}_{2}\right)=\frac{e^{2}}{\varepsilon \sqrt{\left(z_{1}-z_{2}\right)^{2}+4 R^{2} \sin ^{2}\left(\frac{\varphi_{1}-\varphi_{2}}{2}\right)}}$,

respectively. Similarly to the previous case, the Coulomb interaction matrix elements depends on the difference $v_{\mathrm{j}}-v_{\mathrm{i}}$. Therefore, in this case, the energy of the relative motion of a $X^{-}$state is a periodic function of $\Phi$ with a period of $\frac{1+2 \sigma}{1+\sigma} \phi_{0}$. Eqs. (31) and (32) correspond to a transformation of $N_{1} \rightarrow N_{1}{ }^{\prime}=N_{1}-p, N_{2} \rightarrow N_{2}{ }^{\prime}=N_{2}-p$, and $v_{\mathrm{c} . \mathrm{m} .} \rightarrow v_{\mathrm{c} . \mathrm{m}}{ }^{\prime}=v_{\mathrm{c} . \mathrm{m}}$. from Eqs. (27) and (28), and $v_{\text {c.m. }}$ is constant in changing $\Phi$. This extra constraint on $v_{\text {c.m. }}$, which is absent in the case of the previous consideration on Eq. (14) using the electrons and hole coordinates, is the source of the period of $\mathrm{AB}$ oscillation of $\frac{1+2 \sigma}{1+\sigma} \phi_{0}$.

In the second case, $E_{\mathrm{KE}}^{(\mathrm{X}, \text { rel) }}$ is invariant by a transformation of

$\Phi \rightarrow \Phi^{\prime}=\Phi+p \phi_{0}$

$v_{1} \rightarrow v_{1}^{\prime}=v_{1}-\frac{1+\sigma}{1+2 \sigma} p$

and

$v_{2} \rightarrow v_{2}^{\prime}=v_{2}-\frac{1+\sigma}{1+2 \sigma} p$.

Eqs. (36) and (37) correspond to a transformation of $N_{1} \rightarrow N_{1}{ }^{\prime}=N_{1}-p, N_{2} \rightarrow N_{2}{ }^{\prime}=N_{2}-p$, and $v_{\text {c.m. }} \rightarrow v_{c . m .}{ }^{\prime}=v_{c . m}-p$. As in the first case, because the Coulomb interaction matrix elements are invariant by this transformation, the energy of the relative motion of a $\mathrm{X}^{-}$state is a periodic function of $\Phi$ with a period of $\phi_{0}$ if the constraint on $v_{c . m}$. is removed, in agreement with the previous argument using the electrons and the hole coordinates.

In numerically calculating the ground state energy of $\mathrm{X}^{-}$, the wave functions Eqs. (23) and (25) are used. The number of the basis is further reduced by separating the Hilbert space into the singlet and the triplet $\mathrm{X}^{-}$subspaces $[4,15]$. In the followings, we present results for the singlet $\mathrm{X}^{-}$state,

$\left.\Psi^{(\mathrm{X}, \text { rel }}\right)\left(z_{1}, \phi_{1}, z_{2}, \phi_{2}\right)=\sum_{v_{1}, v_{2}} B_{z_{1}, v_{1}, z_{2}, v_{2}}\left\{\frac{1}{\sqrt{2}}\left[\chi_{v_{1}}\left(\phi_{1}\right) \chi_{v_{2}}\left(\phi_{2}\right)+\chi_{v_{1}}\left(\phi_{2}\right) \chi_{v_{2}}\left(\phi_{1}\right)\right]\left(1-\delta_{v_{1} v_{2}}\right)+\chi_{v_{1}}\left(\phi_{1}\right) \chi_{v_{2}}\left(\phi_{2}\right) \delta_{v_{1} v_{2}}\right\}$

which is the ground state in the magnetic field of our interests. 


\subsection{Higher-order finite difference method on generalized grid points}

In the followings, we use units of $a_{\mathrm{B}}^{*}=\varepsilon \hbar^{2} / \mu^{*} e^{2}$ for length and $E_{\mathrm{Ry}}^{*}=\mu^{*} e^{4 / 2} \varepsilon^{2} \hbar^{2}$ for energy. The coordinate along the axis direction is discretized with a generalized grid point $z_{j}^{n}$, where $n$ is an integer in the range of $-n_{\max } \leq n \leq n_{\max }$. The grid points $z_{j}^{n}$ is given by $z_{j}^{n}=\sum_{q=0}^{n} \frac{1}{2}\left(b_{q-1}+b_{q}\right)$ for $n>0, z_{j}^{n}=-\sum_{q=0}^{|n|} \frac{1}{2}\left(b_{q-1}+b_{q}\right)$ for $n<0$, and $z_{j}^{0}=0$. The derivatives with respect to $z_{\mathrm{j}}$ are expanded in real-space by the higher-order finite difference method [18-20] as given by

$$
\left.\frac{\mathrm{d}^{k}}{\mathrm{~d} z_{j}^{k}}\right|_{z=\xi} \approx \sum_{j=0}^{N_{F D}+1} c_{N_{F D}, j}^{k} f\left(z_{j}^{n}\right)
$$

where $\xi=z$ is a point at which the approximations are calculated, $k$ is the order of derivative of interests, and $N_{\mathrm{FD}}$ is the order of the finite difference. A fast algorithm for calculating the coefficients $c_{N_{F D}, j}^{k}$ can be found elsewhere [18].

The attractive and repulsive Coulomb interaction terms are evaluated by numerically integrate on the grid points

$$
g\left(z_{i}-z_{i}, \varphi_{j}-\varphi_{j^{\prime}}\right)=\frac{e^{2}}{\varepsilon \sqrt{\left(z_{i}-z_{i}\right)^{2}+4 R^{2} \sin ^{2}\left(\frac{\varphi_{j}-\varphi_{j^{\prime}}}{2}\right)}},
$$

for $i j \neq i{ }^{\prime} j^{\prime}$, and for $i j=i^{\prime} j^{\prime}$, where logarithmic singularity is present, an explicit integration over the cell is performed,

$$
g(0,0)=\frac{2 e^{2} 1}{\varepsilon b_{0}}\left[\ln \left(\sqrt{t^{2}+1}+1\right)+\frac{\ln \left(t+\sqrt{t^{2}+1}\right)}{t}-\ln (t)\right]
$$

where $t=2 \pi \eta / b_{0}, \eta$ and $b_{0}$ are the grid spacings in the circumference and the axis directions at the origin, respectively. Good numerical accuracy is obtained for $t \sim 1$ and we choose $\eta=\pi / 30$. The integrals with respect to $\varphi$ are performed numerically on a grid spacing of $\eta$. In order to choose an optimal distribution of $z_{j}^{n}$, we first calculate the binding energy of a neutral exciton on a $2 \mathrm{D}$ plane, where an analytical solution is available [21]. Because of the huge Hilbert space required for calculating the energy of $\mathrm{X}^{-}$states, our objective here is to obtain good accuracy of the energy with a minimal basis set. We chose $b_{n}=\pi / 30$ for $0 \leq n \leq 7, \pi / 15$ for $8 \leq n \leq 10,2 \pi / 15$ for $11 \leq n \leq 13$, and $4 \pi / 15$ for $14 \leq n \leq 15$, and $8 \pi / 15$ for $16 \leq n$. The states up to the 
cut-off energy of $E_{z}^{\text {(cutoff) }}=4 \frac{\hbar^{2}}{2 \mu^{*}} \frac{1}{\left(\frac{\pi}{30}\right)^{2}}=365$ are effectively taken into account. The relative motion in the axis direction was truncated at a finite size $L=15.4$. The order of the finite difference $N_{\mathrm{FD}}$ was fixed to 4 except for the grid points near the origin $n=-1,0$, and 1 , where we found that $N_{\mathrm{FD}}=2$ gives better numerical accuracy. With the above parameters, we obtained the 2D exciton binding energy of 4.03, which is compared with the exact value of 4 . In what follows, the lowest energy of the $\mathrm{X}^{0}$ and $\mathrm{X}^{-}$states are calculated with the above parameters except otherwise denoted. Because we are interested in the ground state $K \sim 0, L$ may be interpreted as the length of the tube. We use $\sigma=1 / 4$ for typical III-V semiconductors such as GaAs, InP, or InAs. The angular momentum $N_{\mathrm{j}}$ is truncated to include the basis in the range of $N_{j}^{\min } \leq N_{j} \leq N_{j}^{\max }$, where $\left|N_{j}^{\max }{ }_{-} N_{j}^{\min }\right|$ is set to be 20, 40,60 for $R=0.5,1$ and 1.5, respectively, for a $\mathrm{X}^{0}$ state by keeping the circumference length divided by $\left|N_{j}^{\max }{ }_{-} N_{j}^{\min }\right|$ constant. $N_{j}^{\max }$ and $N_{j}^{\min }$ which give minimum eigenenergy are selected by performing calculations with $\left(N_{j}^{\max }{ }_{-} N_{j}^{\min }\right) / 2$ varied. The cut-off energy in the circumference motion at $\Phi=0$ is $E_{\varphi}^{\text {(cutoff) }}=\frac{1}{1+\sigma} \frac{\hbar^{2}}{2 \mu^{*} R^{2}} 10^{2}=320$. For a $X^{-}$state, $\left|N_{j}^{\max } N_{j}^{\text {min }}\right|$ is set to be 20 for any $R$ due to the limitation of our computational resources. The energy of the relative motion of $\mathrm{X}^{-}$state was found to be overestimated by 0.0111 for a small system of $L=0.94$ and $R=1$ by comparing the results between the cases of $\left|N_{j}^{\max }{ }_{-} N_{j}^{\min }\right|=20$ and 40 . The error in the amplitude of the oscillation was found to be smaller than 0.003 . The lowest state energy and the wave function are obtained by a conjugate gradient method (CGM) for minimizing the energy. The standard CGM routine is modified to allow the arbitrary spaced grids. For example, the matrix element of the Hamiltonian for $\mathrm{X}^{0}$ is calculated as given by

$$
\begin{aligned}
& \int_{0}^{2 \pi} \mathrm{d} \varphi_{1} \int_{-L / 2}^{L / 2} \mathrm{~d} z_{1} \Psi^{\left(\mathrm{X}^{0}, \text { rel }\right) *}\left(z_{1}, \varphi_{1}\right) H^{\left(\mathrm{X}^{0}, \mathrm{rl}\right)} \Psi^{\left(\mathrm{X}^{0}, \mathrm{rl}\right)}\left(z_{1}, \varphi_{1}\right) \\
& =\sum_{n=-n_{\max }}^{n_{\max }} \sum_{l=0}^{N_{\varphi}} A_{z_{1}^{n}, N_{1}}^{*} \chi_{N_{1}}^{*}(l \eta) H^{\left(\mathrm{X}^{0}, \text { rel }\right)} A_{z_{1}^{n}, N_{1}} \chi_{N_{1}}(l \eta) \eta b_{n},
\end{aligned}
$$

where $N_{\varphi}$ is the number of the grid points in the circumference as given by $N_{\varphi}=2 \pi R / \eta$. The number of the basis states is 282,975 for calculating the $\mathrm{X}^{-}$states and the size of the Hamiltonian matrix is $282,975^{2}$. 


\section{Results}

\subsection{Neutral exciton}

With decrease in $R$, the kinetic energy and the electron-hole Coulomb energy in the circumference direction increase with $1 / R^{2}$ and $1 / R$, respectively, leading to larger $\mathrm{AB}$ oscillation amplitude. The Coulomb interaction in the axis direction on the other hand increases with decrease in $R$. The $R$ dependence of the lowest state energy of $\mathrm{X}^{0}$ is shown in Fig. 1. For small $R, \Phi$ dependence of the lowest state energy of $\mathrm{X}^{0}$ is dominated by the single-particle energies of an electron $\frac{1}{1+\sigma} \frac{\hbar^{2}}{2 \mu^{*} R^{2}}\left(N^{(e)}-\frac{\Phi}{\phi_{0}}\right)^{2}$ and a hole $\frac{\sigma}{1+\sigma} \frac{\hbar^{2}}{2 \mu^{*} R^{2}}\left(N^{(h)_{+}} \frac{\Phi}{\phi_{0}}\right)^{2}$, where $N^{(\mathrm{e})}$ and $N^{(\mathrm{h})}$ are the angular momentum quantum numbers for an electron and a hole, respectively. The lowest state energy of $\mathrm{X}^{0}$ shows a sinusoidal oscillation with a period of $\phi_{0}$. The minima of the lowest state occur at integer flux quanta.

The amplitude of the oscillation of the lowest state energy of $\mathrm{X}^{0}, \Delta E^{(\mathrm{x} 0)}=E^{(\mathrm{x} 0)}\left(\Phi=0.5 \phi_{0}\right)$ $-E^{(\mathrm{x} 0)}(\Phi=0)$, is shown in Fig. 2 as a function of $L$ for $R=0.5$. The amplitude of the oscillation rapidly increases with increase in $L$ because of the reduction of the Coulomb interaction and saturates at about $L \sim 4$. This shows that a quantum tube is more favorable for the observation of the oscillation of the $\mathrm{X}^{0}$ energy with $\Phi$ than a quantum ring where the vertical motion is restricted by strong confinement.

\subsection{Charged exciton}

The calculated ground state energies of the relative motion of $\mathrm{X}^{-}$for $R=0.5,1$, and 1.5 are shown in Fig. 3 for the case of center-of-mass angular momentum $v_{\text {c.m. }}$. fixed to zero and for the case of $v_{\mathrm{c} . \mathrm{m}}$. which gives the minimum energy. As shown in Sec. 2.2, the period of AB oscillation of the energy of the relative motion of $X^{-}$is $\frac{1+2 \sigma}{1+\sigma} \phi_{0}=\frac{6}{5} \phi_{0}$ for the former case by substituting $\sigma=1 / 4$, and $\phi_{0}$ for the latter case. In both the cases, $v_{c . m}$. is 0 between $\Phi=0$ and $0.5 \phi_{0}$. A level-crossing of the energy of the center-of-mass motion occurs at $\Phi=0.5 \phi_{0}$. Between $\Phi=0.5 \phi_{0}$ and $\phi_{0}$, the calculated ground state energies of the relative motion is lower for the case of $v_{\mathrm{c} . \mathrm{m} .}=-1$ than for the case where $v_{\mathrm{c} . \mathrm{m} \text {. }}$ is fixed to 0 . 
Upon the optical transition an electron and a hole recombine, leaving an electron in the final state. The optical transition energy of $\mathrm{X}^{-}$as given by $E^{(\mathrm{X}-)}=E_{\mathrm{rel}}+E_{\mathrm{c} . \mathrm{m} .}-E_{\mathrm{sp}}$ is shown in Fig. 4, where $E_{\text {rel }}, E_{\mathrm{c} . \mathrm{m}}$, and $E_{\mathrm{sp}}$ are the energies of the electron-hole relative motion, the center-of-mass motion, and the single electron in the final state, respectively. In Fig. 4, the optical transition energies are shown for the case where no constraint is imposed on $v_{\text {c..m. }}$. As discussed previously, the angular momentum of the lowest state of the center-of-mass motion and the single electron is 0 between $\Phi=0$ and $0.5 \phi_{0}$, and -1 between $\Phi=0.5 \phi_{0}$ and $\phi_{0}$. $E_{\text {c.m. }}-E_{\text {sp }}$ is minima at $\Phi=0.5 \phi_{0}$, while $E_{\text {rel }}$ is maxima. The plots of the optical transition energy of $\mathrm{X}^{-}$change from downward to upward cusp with decrease in $R$. In the intermediate case of $R=0.5$, a double maxima structure in the optical transition energy is seen in Fig. 4 because of the difference in the $\Phi$ dependencies between the single electron energy and the relative motion energy of a $\mathrm{X}^{-}$state.

The amplitude of the oscillation of $\mathrm{X}^{-}$ground state energy of the relative motion $\Delta E^{(\mathrm{X}-)}=$ $E^{(\mathrm{X}-)}(\Phi=0.5)-E^{(\mathrm{X}-)}(\Phi=0)$ is shown in Fig. 5(a) as a function of $R$ for $L=15.4$. The amplitude of the oscillation closely follows the relation $\exp (-C R)$, where $C$ is a constant. This is in agreement with the result in the literature [2] that the amplitude of the oscillation is proportional to $\exp \left(-2 \pi^{2}\left|V_{0}\right| / E_{0}\right)$, where $V_{0}$ and $E_{0}$ are the Coulomb interaction and the kinetic energies, respectively. $\left|V_{0}\right|$ and $E_{0}$ are proportional to $1 / R$ and $1 / R^{2}$, respectively, giving $\exp (-$ $\left.2 \pi^{2}\left|V_{0}\right| / E_{0}\right) \sim \exp (-C R)$. This difference in the $R$ dependencies between the amplitude of the oscillation of the relative motion and the kinetic energy explains the change of the optical transition energy of $\mathrm{X}^{-}$from minima to maxima at $\Phi=0.5 \phi_{0}$ with decrease in $R$ as shown in Fig. 4.

$L$ dependence of $\Delta E^{(\mathrm{X}-)}$ is shown in Fig. 5(b) for $R=0.5$. The amplitude of the oscillation increases with the increase in $L$. This shows that a quantum tube is more favorable for the observation of the oscillation of the $\mathrm{X}^{-}$energy with $\Phi$ than a quantum ring. It is suggested to choose a quantum tube longer than 5 for the observation of the excitonic $\mathrm{AB}$ effect.

The amplitude of the wave function in the axis direction at $\varphi_{1}=0$ is shown in Fig. 6 for the case of $R=0.5$ and $L=15.4$. For a $\mathrm{X}^{0}, \Psi^{\left(\mathrm{X}^{0}, \text { rel }\right)}\left(z_{1}, \varphi_{1}=0\right)$ is plotted. For a $\mathrm{X}^{-}$, a probability function $P\left(z_{1}\right)$ is obtained by integrating over $z_{2}$ and $\phi_{2}$ as given by 


$$
P\left(z_{1}\right)=\int_{-L / 2}^{L / 2} \mathrm{~d} z_{2} \int_{-\pi}^{\pi} \mathrm{d} \varphi_{2} \Psi^{(X ; \text {, rel }) *}\left(z_{1}, \varphi_{1}=0, z_{2}, \varphi_{2}\right) \Psi^{(X ; \text { rel })}\left(z_{1}, \varphi_{1}=0, z_{2}, \varphi_{2}\right)
$$

and $\sqrt{P\left(z_{1}\right)}$ is plotted in Fig. 6. Figure 6 shows that the wave function of $\mathrm{X}^{-}$is extended more in the axis direction than the wave function of $\mathrm{X}^{0}$.

\section{Discussions}

The amplitude of the oscillation saturates at $L=4 \sim 5$ as shown in Figs. 2 and 5. This is understood by the vanishing amplitude of the wave function as shown in Fig. 6 at $\left|z_{1}\right|=2 \sim$ 2.5. The length $L$ at which the amplitude of the oscillation saturates is larger for a $\mathrm{X}^{-}$than a $\mathrm{X}^{0}$, reflecting the more extended wave function for $\mathrm{X}^{-}$in the axis direction. The larger amplitude of the oscillation at $L>6$ for a $\mathrm{X}^{-}$than for a $\mathrm{X}^{0}$ is similarly understood by smaller mixing of the angular momentum due to the larger extent of the wave function for $\mathrm{X}^{-}$than that for $\mathrm{X}^{0}$.

In the limit of vanishing $L$, the system approaches to a $1 \mathrm{D}$ ring. In this case, because the binding energy of the exciton diverges if a long range Coulomb interaction is assumed, the amplitude of the oscillation approaches to zero as $L \rightarrow 0$ as shown in Figs. 2 and 5 . In order to avoid the divergence of the binding energy, Römer and Raikh used a short ranged interaction [2] and Korkusinski et al. introduced a parameter to account for a finite thickness [4], and they obtained a finite amplitude of the oscillation.

A qualitative difference of the $\mathrm{AB}$ oscillation of a $\mathrm{X}^{0}$ and a $\mathrm{X}^{-}$lies in the $R$ dependence of the sign of the oscillation of the optical transition energy. The optical transition energy of a $\mathrm{X}^{0}$ is always maxima at half integer flux quanta, while that of a $\mathrm{X}^{-}$changes from minima to maxima at half integer flux quanta with decrease in $R$. The other important difference between a $\mathrm{X}^{0}$ and a $\mathrm{X}^{-}$is the amplitude of $\mathrm{AB}$ oscillation of the optical transition energy, which is 0.0005 and $0.16\left(E_{\mathrm{Ry}}{ }^{*}\right)$ for a $\mathrm{X}^{0}$ and a $\mathrm{X}^{-}$, respectively, at $R=1$. Experimental observation of $\mathrm{AB}$ oscillation of a $\mathrm{X}^{-}$is much more feasible than that of a $\mathrm{X}^{0}$.

In the previous section, the energy of the relative motion of $\mathrm{X}^{-}$is calculated for the two cases, namely, for fixed $v_{c . m}$. and for $v_{c . m}$. varying with $\Phi$. In usual experimental conditions, the lowest energy of the $\mathrm{X}^{-}$energy is probed at each $\Phi$ after energy relaxation to the ground 
state by inelastic scatterings accompanied with change in $v_{\text {c.m. }}$. However, in a carefully prepared experimental condition, it would be possible to fix $v_{\mathrm{c} . \mathrm{m}}$. while changing $\Phi$ at a sweep rate faster than the angular momentum relaxation rate of the center-of-mass motion. In this case, the period of $\mathrm{AB}$ oscillation would be $\frac{1+2 \sigma}{1+\sigma} \phi_{0}$. The other important case often encountered in experiments is the case where the hole is localized at a fixed position. In this case, because $m \mathrm{e} / m_{\mathrm{h}}=\sigma$ is 0 in the limit of $m_{h} \rightarrow \infty$, the period of $\mathrm{AB}$ oscillation is $\phi_{0}$. Our results can also be applied to this case by setting $E_{\mathrm{c} . \mathrm{m} \text {. }}$ to 0 .

A depolarization field was investigated in carbon nanotubes to weaken the optical absorption peak when an optical electric field is perpendicular to the axis [9]. The carbon nanotubes were assumed to be suspended in vacuum, while we assume that quantum tubes are embedded in matrices with the same dielectric constant $\varepsilon$ as that of the quantum tubes. Therefore, the depolarization effect is not as important in our system.

Typical cylindrical nanostructures are fabricated such as by embedding InP layer in GaAs matrix, InAs layer in InP matrix, or $\operatorname{In}_{x} \mathrm{Ga}_{1-\mathrm{x}}$ As in GaAs matrix [22]. In these cases, the well layers are compressibly strained. The heavy- and light hole bands are mixed by the strain. In the case of relatively large splitting of the hole bands, the effective mass of the upper hole band in a cylindrically symmetric system along the circumference direction is given by $m_{h}=m_{0} /\left(\gamma_{1}-\sqrt{3} \gamma_{2}\right)$ [22], where $\gamma_{1}$ and $\gamma_{2}$ are the Luttinger's parameters. For GaAs well layer, $m_{\mathrm{e}}=0.0665 m_{0}, \gamma_{1}=7.65, \gamma_{2}=2.41$, and $\varepsilon=12.52 \varepsilon_{0}$ give $\sigma=0.23, a_{\mathrm{B}} *=12.2 \mathrm{~nm}$, and $E_{\mathrm{Ry}}{ }^{*}$ $=4.7 \mathrm{meV}$. For InAs well layer, $m_{\mathrm{e}}=0.023 m_{0}, \gamma_{1}=19.67, \gamma_{2}=8.37$, and $\varepsilon=15.15 \varepsilon_{0}$ give $\sigma=0.12, a_{\mathrm{B}} *=39 \mathrm{~nm}$, and $E_{\mathrm{Ry}}{ }^{*}=1.2 \mathrm{meV}$. For InP well layer $m_{\mathrm{e}}=0.079 m_{0}, \gamma_{1}=5.15, \gamma_{2}=$ 0.94 , and $\varepsilon=12.6 \varepsilon_{0}$ give $\sigma=0.28, a_{\mathrm{B}}{ }^{*}=13.0 \mathrm{~nm}$, and $E_{\mathrm{Ry}} *=3.7 \mathrm{meV}$ [23]. The magnetic field $B$ corresponding to $\phi_{0}$ is given by $13.2-0.5 \mathrm{~T}$ for $R=10-50 \mathrm{~nm}$. The $\mathrm{AB}$ oscillation amplitude of the lowest optical transition energy of a $\mathrm{X}^{-}$state is of the order of $1\left(E_{\mathrm{Ry}}{ }^{*}\right)$ for $R=0.25$. This is within the range of detection with currently available samples in the literature [17]. Such measurements are under investigations. 


\section{Conclusions}

$\mathrm{AB}$ oscillations of the optical transition energies of $\mathrm{X}^{0}$ and $\mathrm{X}^{-}$in a quantum tube are investigated as a function of $R$ and $L$. The amplitudes of the oscillation of the relative motion of the ground state energies are shown to increase with decrease in the restriction of the motion parallel to the axes of a quantum tube, showing that a quantum tube is more favorable than a quantum ring for observation of the excitonic $\mathrm{AB}$ effect. The energy of the relative motion of a $\mathrm{X}^{-}$state is shown to be a periodic function of $\Phi$ with a period of $\phi_{0}$ for the case where no constraint is imposed on the angular momentum of the center-of-mass motion $v_{\text {c.m. }}$ and $\frac{1+2 \sigma}{1+\sigma} \phi_{0}$ for the case where $v_{\text {c.m. }}$. is constant in changing $\Phi$. We find a double maxima structure in the optical transition energy for $R=0.5$ because of the difference in the $\Phi$ dependencies between the single electron energy and the relative motion energy of a $\mathrm{X}^{-}$ state.

\section{Acknowledgements}

Part of this work was supported by Grant-in-Aid for Scientific Research by Japan Society for the Promotion of Science, and Nano-Science Special Project by University of Tsukuba. We thank T. Fukui and J. Motohisa for fruitful discussions. 


\section{References}

${ }^{1}$ A. Chaplik, JETP Lett. 62, 900 (1995).

${ }^{2}$ R.A. Roemer and M.E. Raikh, Phys. Rev. B 62, 7045 (2000).

${ }^{3}$ M. Bayer, M. Korkusinski, P. Hawrylak, T. Gutbrod, M. Michel, and A. Forchel, Phys. Rev. Lett. 90, 186801 (2003).

${ }^{4}$ M. Korkusinski, P. Hawrylak, and M. Bayer, Phys. Status Solidi (b) 234, 273 (2002).

${ }^{5}$ E. Ribeiro, A.O. Govorov, J. W. Carvalho, and G. Medeiros-Ribeiro, Phys. Rev. Lett. 92, 126402 (2004).

${ }^{6}$ L. Banyai, I. Galbraith, C. Ell, and H. Haug, Phys. Rev. B 36, 6099 (1987).

${ }^{7}$ T. Ogawa and T. Takagahara, Phys. Rev. B 43, 14325 (1991).

${ }^{8}$ H. Ajiki and T. Ando, J. Phys. Soc. Jpn. 62, 2470 (1993).

${ }^{9}$ H. Ajiki and T. Ando, Physica B 201, 349 (1994).

${ }^{10}$ T. Ando, J. Phys. Soc. Jpn. 73, 3351 (2004).

${ }^{11}$ T. Nakanishi and T. Ando, J. Phys. Soc. Jpn. 74, 3027 (2005).

${ }^{12}$ G. Munschy and B. Stebe, Phys. Status Solidi (b) 64, 213 (1974).

${ }^{13}$ G. Finkelstein, H. Shtrikman, and I. Bar-Joseph, Phys. Rev. Lett. 74, 976 (1995).

${ }^{14}$ A. Wojs and P. Hawrylak, Phys. Rev. B 51, 10880 (1995).

15D.M. Whittaker and A.J. Shields, Phys. Rev. B 56, 15185 (1997).

16A.B. Dzyubenko and A.Y. Sivachenko, Phys. Rev. Lett. 84, 4429 (2000).

17P. Mohan, J. Motohisa, and T. Fukui, Appl. Phys. Lett. 88, 133105 (2006).

18B. Fornberg and D.M. Sloan, in Acta Numerica 1994, pp. 203 ed. A. Iserles (Cambridge University Press, Cambridge, 1994).

19J.R. Chelikowsky, N. Troullier, and Y. Saad, Phys. Rev. Lett. 72, 1240 (1994).

${ }^{20}$ S. Nomura and Y. Aoyagi, Phys. Rev. Lett 93, 096803 (2004).

${ }^{21}$ M. Shinada and S. Sugano, J. Phys. Soc. Jpn. 21, 1936 (1966).

${ }^{22}$ K. Nishi, A.A. Yamaguchi, J. Ahopelto, A. Usui, and H. Sakaki, J. Appl. Phys. 76, 7437 (1994).

${ }^{23}$ Landolt-Börnste in Numerical Data and Functional Relationship in Science and Technology, New Series, Group III, Vol. 17 pt. a (Springer-Verlag, Berlin, 1982). 


\section{Figure captions}

Figure 1 Ground state energies of $\mathrm{X}^{0}$ for $R=1.5$ (i), 1 (ii), and 0.5 (iii).

Figure $2 \mathrm{AB}$ oscillation amplitude of $\mathrm{X}^{0}$ for $R=0.5$ as a function of $L$.

Figure 3 Ground state energies of the relative motion of $\mathrm{X}^{-}$for (a) $R=1.5$, (b) 1 , (c) 0.5 for the cases of (i) $v_{\text {c.m. }}=0$ at $0 \leq \Phi \leq 0.5 \phi_{0}$ and $v_{\text {c.m. }}=-1$ at $0.5 \phi_{0} \leq \Phi \leq \phi_{0}$, giving the minimum energy, and (ii) $v_{\text {c.m. }}=0$.

Figure 4 Magnetic flux dependence of $E_{\mathrm{c} . . \mathrm{m} .} R^{2}$ (open circles) (i), $-E_{\mathrm{sp}} R^{2}$ (open squares) (ii), $E_{\text {rel }}$ for $R=1$ (solid squares) (iii), $E\left(\mathrm{X}^{-}\right)=E_{\mathrm{c} . . \mathrm{m} .}+E_{\mathrm{rel}}-E_{\mathrm{sp}}$ for $R=1$ (solid circles) (iv), $E\left(\mathrm{X}^{-}\right)$ for $R=0.5$ (solid circles) (v), and $E\left(\mathrm{X}^{-}\right)$for $R=0.25$ (solid circles) (vi).

Figure 5 (a) Amplitude of the oscillation of $X^{-}$ground state energy of the relative motion for $L=15.4$ as a function of $R$ (solid circles). The solid line is the best fitted line. (b) AB oscillation amplitude of $\mathrm{X}^{-}$for $R=0.5$ as a function of $L$.

Figure 6 Amplitude of the wave function of (a) $X^{0}$ and (b) $X^{-}$at $\varphi_{1}=0$ as a function of $z_{1}$ for $R=0.5$. 